NOTICE WARNING CONCERNING COPYRIGHT RESTRICTIONS:

The copyright law of the United States (title 17, U.S. Code) governs the making of photocopies or other reproductions of copyrighted material. Any copying of this document without permission of its author may be prohibited by law. 


\title{
ABSTRACTION and VERIFICATION in ALPHARD: A Symbol Table Example
}

\author{
Ralph L. London, USC Information Sciences Institute \\ Mary Shaw, Carnegie-Mollon University \\ Wm. A. Wulf, Carnegie-Mellon University
}

December 29, 1976

Abstract: The design of the Alphard programning language has been strongly influenced by ideas from the areas of programming methodology and formal program verification. In this paper we design, implement, and verify a general symbol table mechanism. This example is rich enough to allow us to illustrate the use as well as the definition of programmer-defined abstractions. The verification illustrates the power of the form to simplify proofs by providing strong specifications of such abstractions.

Keywords and Phrases: abstraction and representation, abstract data types, assertions, corroctness, data abstraction, data structures, information hiding, modular decomposition, program specifications, program verification, programming methodology, structured programming

The research described here was supported in part by the National Science Foundation (Grant DCR74-04187) and in part by the Defense Advanced Research Projects Agency (Contracts: F44620-73-C-0074, monitored by the Air Force Office of Scientific Research, and DAHC-1572-C-0308). The views expressed are those of the authors. This report is one in a series being printed jointly by CMU and ISI. 
Page 2

\section{Contents}

Introduction--The Symbol Table Task .....................................................

The Symbol Table Abstraction ..................................................................... 5

Implementation of Symbol Table ...............................................................

Verification of the form Symtab ................................................................. 12

Implementation of the form Condis ........................................................

Verification of Condis .......................................................................... 19

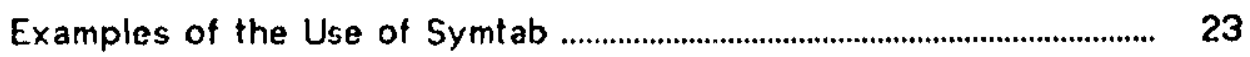

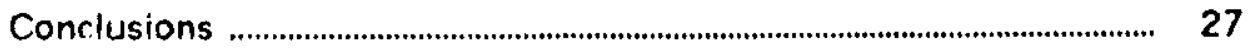

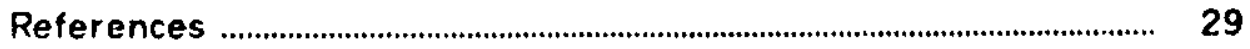

Appendix A: Informal Description of Verification Methodology ......... 30 


\section{Introduction---The Symbol Table Task}

Previous reports [Shaw76b, Wulf76a,b] have described the Alphard programming language and its associated verification methodology. These reports developed Alphard definitions for the canonical examples of data abstractions (stacks, queues, and sets). These examples are sufficiently simple to be grasped readily, and they have appeared often enough in other languages that the reader may compare various approaches to their definition. There is, however, a danger in considering only these examples. It is possible that an approach will work for only the easy examples, or that the definition of something more complex will be far less elegant.

Therefore, in this report we shall consider a larger, more realistic example: an abstraction of a symbol table. For comparison purposes the reader may wish to refer to the similar example given in [Guttag 76] and to a hashtable example in [Wegbreit76]

Suppose that we must produce a number of compilers, assemblers, and interpreters to operate on several different computers. Each such system will contain a symbol table mechanism; although each system will have its own requirements, many of the gross, abstract properties of these symbol tables will be the same. It seems desirable to have a single implementation of these common aspects which is verified; that will be our aim.

But what are the common properties? Many texts [e.g., Gries71] describe a symbol table as a mapping from identifiers (strings appearing in a source program) to a set of attributes associated with those identifiers. Examples of such attributes include "type", "runtime memory address", "number of dimensions" (for arrays), etc. In some cases, the mapping may be sensitive to the context in which the identifier occurs. (Algol-like block structure is the most common example of this context sensitivity; the mapping from identifier to attributes depends upon the block in which the identifier appears. Name qualification, as in field selection from a record, is another example in which the interpretation of the field selector depends upon the type of the record.) The common properties, then, are ones which involve the application and manipulation of this mapping; principally

- some means to apply the mapping, i.e., to find the attributes associated with the occurrence of an identifier.

- some means to alter the mapping, e.g., by inserting and/or deleting entries and signaling changes in context.

Since we want our abstraction to serve a spectrum of languages, system types (e.g., compilers and assemblers), and machines, it would not be appropriate to include the specific attributes as part of the abstraction. Rather, we shall presume that the user of our 
abstraction will define some mechanism for storing and retrieving attributes, e.g., a vector of records; our abstraction will then provide a mapping from an identifier to a unique integer which, for example, may then be used as an index into this vector of attribute records.

Concerning the issue of context sensitivity, we shall provide an abstraction which supports block structure because (1) it is the more general case and (2) with proper implementation, the generality costs very little when it is not used. We shall not explicitly provide for the kind of context sensitivity needed for record selectors, but we shall show how the abstraction may be used to achieve it.

Note that the informal lerm "block-structured" does not describe a unique name-binding policy. For example, consider the program fragment

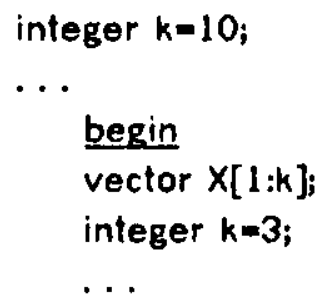

In the declaration of the vector " $X$ ", there is a question about which " $k$ " should be used to define its upper bound. The semantics of some languages specify that the value of the variable " $k$ " defined at the outer block level, i.e., 10, should be used; other languages specify that it is the innermost definition, i.e., "integer $k=3$ ", which should be used. To accommodate the second of these schemes requires that a full lexical analysis pass be performed before any name binding (symbol table construction) is done.

In order to make our abstraction useful on this pure lexical pass, as well as later when the full symbol table is constructed, we shall define it as a mapping between "things" and integers. In a simple system the "things" will be identifiers and the integers will probably be indices into the vector of attributes described above. In a more complex system, the initial lexical pass may use the abstraction to convert identifiers into integers; these integers may in turn be the "things" mapped into symbol table indices during a later pass. An example of the use of the abstraction will be given later to help clarify this point; for the moment the reader may simply assume that the "things" are identifiers.

Summarizing, then, our abstraction shall provide:

(a) A block-structured mapping from "things" to integers.

(b) A set of six operations to insert a new "thing", to lookup the integer associated with a specific "thing", to test whether a specific "thing" is defined at the current block level, to enter and to loave block level, and to 
test whether the mapping is full, i.e., whether there is room for another "thing".

\section{The Symbol Table Abstraction}

The preceding section provides an informal description of the symbol table abstraction; in this section we shall be more precise. Specifically, the specifications part of the form called "symtab" is: 1

form symtab(T:form< $\leftarrow,-$, ,hash( $T, k$ :integer) returns $x$ :integer pre $(k>0)$ post $(0 \leq x<k$ ') $>$, $m, n:$ integer $)=$

\section{beginform}

specifications

requires $n \geq 1 \wedge m \geq 1$;

let symtab $=\langle$ block:integer, assoc:\{<s:T,bl:integer,ui:integer $>\}>$;

invariant

cardinality (assoc) $\leq n$

$\wedge 1 \leq$ ui $\leq n \wedge 1 \leq$ blsblock

$\wedge\left(t_{1}, t_{2}+\right.$ assoc $\supset\left(t_{1} \cdot s=t_{2} \cdot s \wedge t_{1} \cdot b\left|=t_{2} \cdot b\right|=t_{1}\right.$.uimet $\left.\left.2 \cdot u i\right)\right)$;

initially symtab $=\langle 1,\{\}\rangle$

functions

defined(st:symtab,str:T) returns t:boolean

post $t=\exists i$ st $<$ str, st.block',i> ( st.assoc,

insert(st:syintab,str:T) returns i:integer

pre cardinality(st.assoc) $<n \wedge$-defined(st,str)

post st = <st.block', st.assoc' U \{<str,st.block',i>\}>,

lookup(st:syintab,str:T) returns $x$ integer

post if $\exists y \in$ st.assoc st [y.s=str $\wedge \forall z<$ st.assoc, $z . s=s t r \supset z . b \mid \leq y . b l]$

then $x=y . u i$

else $x=0$,

enterblock(st:symtab)

post st $=\langle$ st.block'+1, st.assoc'>,

leaveblock(st:symtab)

pre st.block $>1$

post st $=\langle$ st.block'-1, st.assoc' $-\{\langle s, x, u i\rangle$ st $x \geq s t$. block' $\}>$,

1 A primed variable (e.g., k') represents the value of that variable prior to the execution of an operation. To shorten the pre, post in and out conditions in our papers, we often, by convention, omit assertions about variables which are completely unchanged. Thus for example, we have omitted st $=$ st' from the post condition of defined. 
full(st:symtab) returns t:boolean

post $t=($ cardinality $($ st. assoc $)=n$ );

Note that, abstractly, a symbol table consists of a pair: an integer, "block", and a set, "assoc". The integer denotes the current block level, has the initial value 1 , and is altered only by the operations enterblock and leaveblock. The set, initially empty, consists of triples containing the "thing" defined, the block level at which it was defined, and the unique integer ("ui") associated with the <thing,block level> pair.

The parameters of the form specify the type, "T" (usually strings), of "things" to be entered in the table, and the maximum number, " $n$ ", of simultaneous entries permitted. The parameter " $m$ " is a bit more difficult to explain, and we shall for a moment defer it, together with the discussion of the required rights of $T$.

Since the symbol table contains only currently defined things, the block level of each entry must be legitimate (e.g., between 1 and the current value of "block"). Further, since a maximum of $n$ entries is allowed, the "associated integer" must lie between 1 and $n$. The let clause and the abstract invariant express these restrictions (the last line of the invariant expresses the uniqueness of the integer associations). The remainder of the specifications states that the initial symbol table has a block level of 1 and an empty "assoc" set, and then lists the symbol table functions and their abstract pre and post conditions.

Now, let us return to the issue of the parameter $m$ and the required rights on $T$. As may be seen from the requires clause of the specifications the only requirement on $m$ is that its value be strictly positive; it does not enter into any of the other parts of the formal specification. Hence, one may properly conclude that its precise value is immaterial and the abstraction will function correctly with any positive value.

The value of $m$ does, however, affect the performance of the abstraction. Neither Alphard nor other languages with similar goals have yet found an appropriate way to specify performance properties. In practical systems, of course, such properties are of paramount importance. Since we now have no formal way of specifying them, we must give a small peek into the representation in order to explain the significance of $\mathrm{m}$. (Indeed, the need to have $\mathrm{m}$ and the hash function name in the specifications has essentially revealed the techniques used in the implementation of the abstraction.) The representation uses a hash table, with collisions resolved by chaining, and $m$ specifies the size of this table, i.e., the number of values that the hash function may assume. Although any positive value of $m$ will work, larger values will tend to provide faster searches at the expense of some additional storage.

In addition, the value of $m$ may affect the distribution of "hits" on any particular hash table entry; see [Knuth73] for a discussion of hashing functions and their properties. We will not discuss these properties here, but note that the form $T$ which defines the things stored in the symbol table is required to provide a hashing function which, given an object of type $T$ 
and an integer $k$, returns an integer in the range 0 to $k-1$. Thus, an appropriate choice of $m$ depends in part on the properties of this function.

\section{Implementation of Symbol Table}

In choosing the implementation of the symbol table abstraction, we have been careful to pick a practical one; it is, in fact, one which is used in several commercial compilers. We chose to do this rather than, for example, to use a direct implementation in terms of sets (e.g., the simpleset form defined in [Shaw 76b]). We have done this in order to emphasize that both the language and verification methodology are intended to be used for practical, production quality systems. The more direct implementation, and also its proof, would have been straightforward and clear. However, it would not have been a production quality implementation and thus would not have been useful in a real system. We shall comment on this point further in the conclusion, but we feel strongly that language, methodology, and verification must respond to the requirements of practical, efficient systems.

We shall obtain the implementation in two steps. We shall define an intermediate abstraction (form) in the process of oblaining the complete implementation. This intermediate abstraction will support a restricted, but not uncommon, style of list-processing.

Now, whenever a system implementation is described, one is faced with a presentation problem: whether the description should be "lop-down" or "bottom-up". Both have advantages. In this case we have chosen to make the presentation predominantly top-down -primarily to emphasize that the implementation of lower level abstractions is irrelevant to the correctness of the higher level ones. The next paragraph, however, is an exception to the predominant flavor of the presentation; it describes the implementation of the symbol table in low-level terms, as it will exist after compilation of the forms. It is included for those of us (including the authors) who still need concrete representations to aid their reasoning; purists may simply skip the next paragraph.

The symbol table will be implemented as a hash table with explicit entries for the symbol and its declaration block level, but an implicit encoding of the integer mapping. Hash collisions are resolved by associating a linked list of symbol table entries with each value of the hash function. Each new entry is inserted at the head of the appropriate list. The entries on the lists are therefore ordered by block level (innermost block first). To find the innermost instance of a symbol, lookup need only perform a linear search of the list associated with the hash value of the symbol; the first instance of the symbol in the list is necessarily the one declared at the innermost block tevel. It is a simple matter for leaveblock to delete the proper entries from the heads of these lists.

The implementation of symtab presumes the existence of a form called "condis" 
(collection of named, disjoint integer sequences). The explanation of the symtab implementation will require that we first understand (i.e., specify) condis. Although condis is intended to support a group of linear lists, its abstract specification is stated in terms of more mathematically tractable entities, namely sets and sequences. ${ }^{2}$ The verification of symtab will use the abstract specification from condis but nothing else. The verification of condis will be independent of symtab and its verification. The specifications part of condis is:

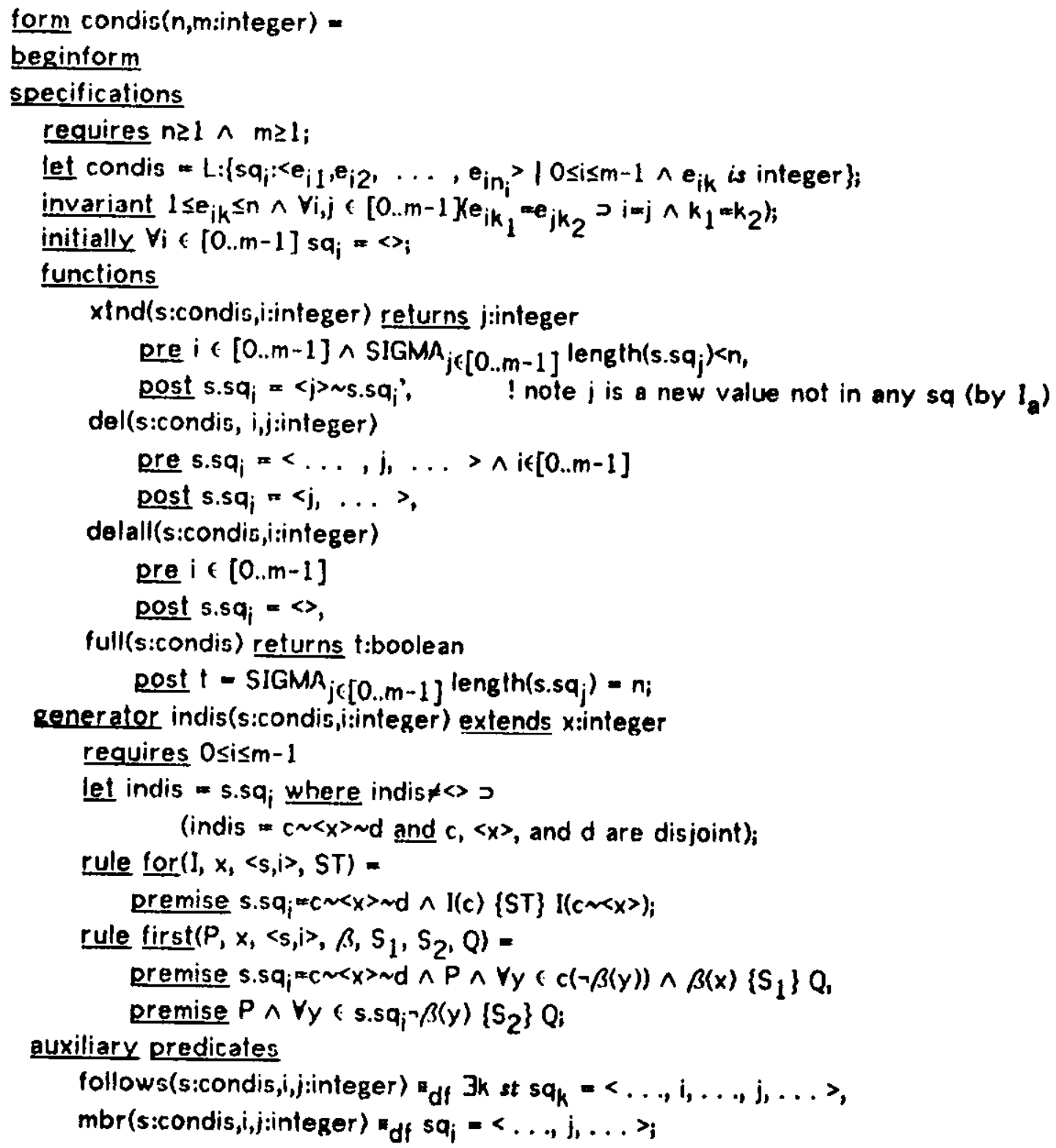

A condis is abstractly described as a set of precisely $m$ sequences of integers; these

2 Definitions and properties of sets appear in [Halmos60] and those of sequences in [Wulf 76a,b]. 
sequences are named $s q_{0}$ through $s q_{m-1}$. The abstract invariant asserts that: (1) each integer in any of the sequences lies in the range 1 to $n$ and (2) a particular integer appears as a sequence element at most once in the entire set of sequences. From these two facts we can observe that the sum of the lengths of the sequences is at most n; moreover, in the case that this sum is $n$, each of the integers 1 through $n$ will appear (precisely once) in one of the sequences.

As a practical matter, each of the sequences in the condis will represent a linear list; specifically, $s q_{i}$ will be associated with the value $i$ produced by the hash function. The sequence elements will be the (integer) indices into a vector of information within symtab; thus the sequence $s q_{i}$ (and the corresponding entries in the vector of information) will represent the linear list of triples in the abstract "assoc" set of symtab which have the hash function value $i$.

Four functions and a generator are provided by the condis form. Function xtend extends the head of a specified sequence by one element; the abstract invariant prevents this integer from being one which already appears in some sequence. Function del permits the initial elements of a specified sequence to be deleted, and function delall permits all the elements of a specified sequence to be deleted. Function full tests whether all of the integers alroady are in some sequence. Generator indis produces the elements of a specified sequence in order, starting with the head. The specification of condis also gives two auxiliary predicates (follows and mbr). These may be used in proofs, but are not actually implemented as executable functions; they should be viewed as an extension to the abstract vocabulary.

At first sight, the condis abstraction may seem unusual; however, we chose to define it in this way for two reasons:

- By using integers to denote elements, we can obtain an efficient encoding of the unique integer mapping required by symtab. This encoding is one which might be selected in actual practice.

- This definition allows us to skirt the issue of pointers (references) for purposes of this paper. 3

Now we can present the complete definition of the symtab form.

3 As most people who have followed the recent literature on programming methodology and verification are aware, the presence of references (unconstrained pointers) in a programming language interferes with our ability to understand and verify programs that use them. While we believe we have made significant progress in Alphard toward resolving the problems introduced by the unconstrained pointer, we will not complicate this paper with pointer issues. 
form symtab(T:form< $\leftarrow,=$, hash(T,k:integer) returns $x$ :integer pre $(k>0)$ post $\left(0 \leq x<k^{\prime}\right)>$, $m, n:$ integer $)=$

beginform

specifications

requires $n \geq 1 \wedge m \geq 1$;

let symtab = <block:integer, assoc:\{<s:T,bl:integer, vi:integer>\}>;

invariant

cardinality (assoc) $\leq n$

$\wedge 1$ suisn $\wedge 1$ bblisblock

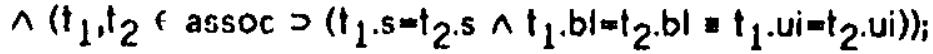

initially symtab $=\langle 1,\{\}\rangle$

functions

defined(st:symtab,str:T) returns t:boolean

post t - Ji st <str,st.block',i> c st.assoc,

insert(st:syintab,str:T) returns i:integer

pre cardinality(st.assoc) $<n \wedge$-defined(st,str)

post st $=\langle$ st.block', st.assoc' $\cup$ \{<str,st.block',i>\}>,

lookup(st:symtab,str:T) returns $x$ :integer

post if $\exists$ $y$ \& st.assoc st [y.s $=$ str $\wedge$ $V z \in$ st.assoc, z.s-str $\supset$ z.bl $\leq y . b l]$

then $x=y$.ui

else $x=0$,

enterblock(st:symtab)

post st = 〈st.block'+1, st.assoc'>,

leaveblock(st:syintab)

pre st.block $>1$

post st $=\langle$ st.block'-1, st.assoc' $-\{\langle s, x$, ui $\rangle$ st $x \geq s t$. block' $\}\rangle$,

full(st:symtab) returns t:boolean

post $t=($ cardinality (st.assoc) $=n)$;

\section{representation}

unique

blvl: integer,

info: vector(record(s:T,bl:integer), $1, n)$,

as: condis $(n, m)$

init blv| $\leftarrow 1$;

$\underline{\text { rep }}(a s, i n f o, b \mid v l)=\langle b|v|,\{<i n f o[i] s, i n f o[i] b|, i>| \exists j \in[0 . . m-1] \text { st } m b r(a s, j, j)\}\rangle_{i}$ invariant

$(m b r(a s, i, j) \supset$ hash(info[j].s, $m)=i)$

$\wedge$ (follows $(a s, i, j) \supset$ blvl $\geq \operatorname{info}[i] b l \geq \operatorname{info}[j] b l \geq 1 \wedge$ (info[i]-info[j] $\supset i-j)$ )

implementation

body defined out $(t=\exists j$ st st.info[j]rr<str,st.b|v|> $\wedge$ mbr(st.as,hash(str,m),j)) -

first j:indis(st.as,hash(str,m)) suchthat st.info[j]swstr

then $t \leftarrow$ st.info[j].bl-st.blvi else $t \leftarrow$ false; 


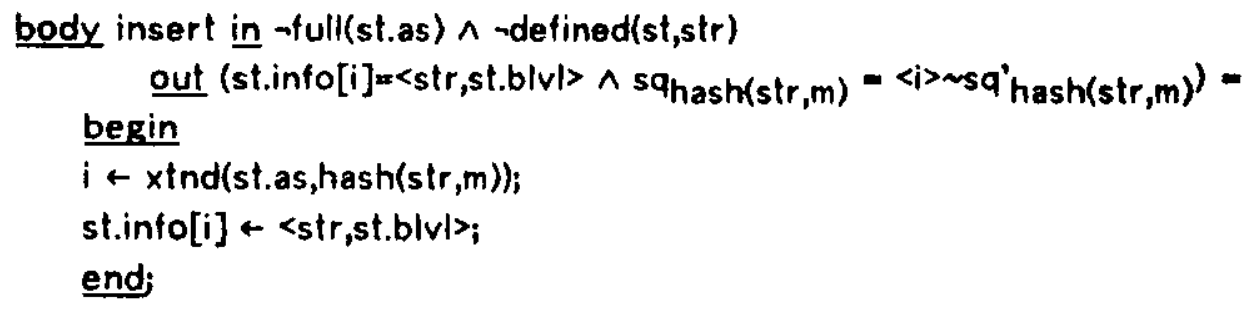

body lookup out $(x=0 \supset(j \in[1 . . n] \wedge \exists i \in[0 . . m-1](m b r(s t . a s, i, j))>$ st.info[j]s $\&$ str $)) \wedge$ $(x>0$ > st.info[ $[x] . s *$ str $\wedge$ (st.info[j].smstr $\supset j=x \vee$ st.info[ $[x] b \mid>$ st.info[j].bl)) $=$ first j:indis(st.as,hash(str,m)) suchthat st.info[j]s-str

then $x \leftarrow j$ else $x \leftarrow 0$;

body enterblock out (st.blvl $=$ st.blvl' +1$)=$

st.blvl $\leftarrow$ st.b|v|+1;

body leaveblock in st.blv| $>1$

out (st.blv| $=$ st.blv|' $-1 \wedge(j \in[1 . . n] \wedge i \in[0 . . m-1]>$

$\left(m b r(s t . a s, i, j)=m b r\left(s t . a s^{\prime},{ }_{i}, j\right) \wedge\right.$ st.info[j].bl $<$ st.b|v|'))) $=$

begin

st.blvl $\leftarrow$ st.blvi-1;

for i: upto $(0, m-1)$ do ! the generator upto is defined in [Shaw76b]

first j:indis(st.as,i) suchthat st.info[j].bl $\leq$ st.blvl

then del(st.as,i,j) else delall(st.as,i);

end;

body full out $\left(t=\operatorname{SIGMA}_{\left.j \in[0 . . m-1]^{\text {length }}\left(s . s q_{j}\right)=n\right)=}\right.$

$t \leftarrow$ full(st.as);

endform

Note that the representation of a symtab consists of three objects: (1) bluh an integer, is a direct representation of the abstract entity block, and is initialized to 1. (2) info is a vector of records which hold the "thing" (usually a string) and the block level at which it was declared. Each of these records is, in effect, one of the triples in the abstract "assoc" set; the third element of the triple, the unique integer, is not explicitly represented -- rather, it is implicitly encoded as the index of this record in the vector. (3) as is a condis, and as explained above, it represents a set of lists of indices into this vector of records; each such list is uniquely associated with a hash function value.

A point which may not be obvious is worth noting. It is rare that all info entries will be in use; we thus have a potential problem in maintaining the free storage of this vector. This problem is handled by the condis abstraction. The uniqueness of the integers in condis sequences guarantees that no info entry will be used simultaneously by different members of assoc. In essence, the integer values which are in the condis sequences correspond to 
occupied entries, and all other integers in the range 1 to $n$ correspond to unoccupied, or free, entries. Specifically, the abstract invariant of condis and the post condition of $x$ tnd together provide a safe allocation of new info entries. Similarly, del and delall provide a safe deallocation mechanism.

To illustrate the operation of the implementation, consider the interaction of the bodies of insert and lookup. When a new symbol is to be inserted, we first invoke the condis operation $x$ tnd. This has the effect of extending the head of the sequence associated with the hash value of the symbol by a new, unique, integer. This integer is then used as the index into the vector info and the symbol and current block level are recorded in this entry. When a later lookup is performed on this symbol, the indis generator is used to find the first integer, $j$, in the sequence associated with the hash value of the symbol for which "info[j].s" matches. Since $x$ tnd extends the sequence at its head, this match is necessarily the most recently declared instance of the symbol.

\section{Verification of the form Symtab}

A torm is verified by proving four properties as described in [Wulf76a,b] and summarized in Appendix A. As promised earlier, the verification below uses only the abstract specification of the form condis, including the auxiliary predicates. The implementation of condis is, as desired, irrelevant to symtab. All uses of the generator indis satisfy the independence assumption provided that in leaveblock we regard both the then and else clauses as being outside the first generator. 4

For the form

1. Representation validity

Show: $I_{c}$ (as,info,blvl) $>I_{a}$ (rep(as,info,blvl))

Proof: cardinality (assoc) $\leq n$ follows from $I_{a}$ for condis, namely, $1 \leq e_{i k} \leq n$ and no duplicate $c_{i k}$ 's means at most $n$ elements in assoc. The relation $1 \leq u i s n$ holds because of mbr in the rep function and $1 \leq e_{i k} \leq n$ in $l_{a}$ for condis. The relation $1 \leq b \mid \leq b l o c k$ follows by setting $j=i$ in follows $(a s, i, j)$ in $l_{c}$. To show uniqueness in assoc, first note that identical $s$ and

4 Strictly speaking, this violates the definition of the first statement in [Shaw76b], a definition which we must modify to permit, for example, finalization statements and the leaveblock usage. We must also weaken the independence assumption. With the strict interpretation, however, an ad hoc argument shows that there are no problems in this case because indis does not modify the generated sequence and no further generation is attempted after the then and else clauses. 
identical bl means, letting hash(t1.s, $m)=$ hash $(t 2 . s, m)=k$, that $m b r(a s, k, t 1 . u i)$ and $\operatorname{mbr}(a s, k, t 2 . u i)$, whence we have either follows(as,t 1.ui,t2.ui) or follows(as,t2.ui,t1.ui). In either case, since info[t1.ui] $=$ rinfo[ $\{2 . u i]$, then $\$ 1$.ui $=\$ 2$.ui as required. The converse of the uniqueness clause holds since $l_{a}$ for condis means no duplicates.

2. Initialization

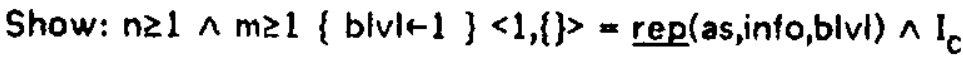

Proof: This holds since initially of condis says each $s q_{j}=<>, i . e,-m b r(a s, j, i)$ and follows $(a s, i, j)$. Note that $n \geq 1 \wedge m \geq 1$ permits the declaration as:condis.

For the function defined

3. Concrete operation

Show: $I_{c}$ (first j:indis(st.as,hash(str,m)) suchthat st.into[j].s-str

then $t \leftarrow$ st.info[j].bl=st.blvl else $t+$ talse $\}$ Aout $\wedge I_{c}$

Proof: $I_{c}$ holds since it is unchanged. Indis may be called since Oshash $(s t r, m)<m$. By the first term of $I_{c}$, str can only be located from $s q_{\text {hash }}(s t r, m)$. For the then clause, the second term of $I_{c}$ gives $\beta_{\text {out }}$. (Note that $\mathrm{mbr}(s t . a s$, hash $(s t r, m), j)$ holds by the definition of indis.) For the else clause str was not located from $s q_{h a s h(s t r, m)}$ whence $t$ is false as required.

4a. $\beta_{\text {in }}$ holds

$\beta_{\text {in }}$ is true

4b. $\beta_{\text {post holds }}$

Show: $I_{c} \wedge \beta_{\text {out }} \supset t=3 i$ st <str,st.block', i> \& st.assoc

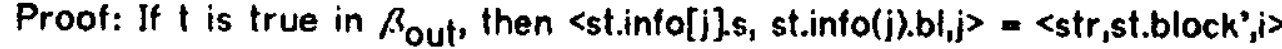
$\epsilon$ st.assoc, i.e., choose $i$ to be $j$. If $t$ is false in $\beta_{\text {out }}$, there will be no $i$ and $t$ is false as required.

For the function insert

3. Concrete operation

Show: $\beta_{\text {in }} \wedge I_{c}\{$ i-xtnd(st.as, hash(str,m)); st.info[i $\}-<$ str,st.blvl> $\} \beta_{\text {out }} \wedge I_{c}$ Proof: The pre of xtnd holds because hash(str,m) $\in[0 . . m-1]$ and because rfull(st.as) means cardinality(st.assoc) $<n$ whence the SIGMA term $<n$. The first term of $\beta_{\text {out }}$ is clear. Since the hash(str, $\left.m\right)^{\text {th }}$ sequence of as is extended, $s q_{\text {hash }}(s t r, m)=$ <i>nsqhash(str,m) where $i$ is the appended new element. The first term of $I_{c}$ follows by the call to $x$ tnd and st.info[i]smstr; the second term of $I_{c}$ follows by $I_{c}$ and rdefined(st,str), i.e., str is not defined at the current block.

4a. $\beta_{\text {in }}$ holds

Show: $I_{c} \wedge$ cardinality(st.assoc) $<n \wedge$ udefined(st,str) $\supset \beta_{\text {in }}$

Proof: cardinality(st.assoc) $<n$ means -full(st.as). 
4b. $\beta_{\text {post holds }}$

Show: $I_{c} \wedge A_{\text {pre }} \wedge A_{\text {out }} \supset \beta_{\text {post }}$

Proof: The new triple <st.info[i]s,st.info[i]bl,i> is added to st.assoc.

For the function lookup

3. Concrete operation

Show: $I_{c}$ ( first jindis(st.as,hash(str,m)) suchthat st.info[j].smstr

then $x+j$ else $x+0\}$ Bout $\wedge I_{c}$

Proof: $I_{c}$ is unchanged. As in the operation defined, str can only be located from sqhash(str,m) By indis, $j \in[1 . . n]$. Hence only the else clause makes $x=0$ and, as required in this case, $j \in[1 . . n] \wedge \exists i \in$ $[0 . . m-1](m b r(s t . a s, i, j))>$ st.info[j].słstr. For the then clause, the first term after $x>0$ holds by the suchthat clause. For the second term after $x>0$, suppose $j \neq x$. Using the second term of $I_{c}$ (note that follows $(s t . a s, x, j)$ holds) rules out the possibility that st.info[ $[x]$.bl=st.info[j].bi since otherwise $j=x$. Hence st.info[ $[x] b \mid>$ st.info[j].bl.

4a. $\beta_{\text {in holds }}$

$\beta_{\text {in }}$ is true

4b. $\beta_{\text {post holds }}$

Show: $I_{c} \wedge \beta_{\text {out }} \supset A_{\text {post }}$

Proof: $x=0$ means $-\exists y$ st $y . s=s t r . ~ x>0$ means $x=y . u i$, i.e., $y=$ <st.info[j].s,st.info[j].bl,j>.

For the function enterblock

3. Concrete operation

Show: $I_{c}\{$ st.blvi + st.blvl+1 $\} \beta_{\text {out }} \wedge I_{c}$

Proof: $\beta_{\text {out }}$ is clear. Since st.blvi increases, $I_{c}$ still holds.

4a. $\beta_{\text {in }}$ holds

$\beta_{\text {in }}$ is true

4b. $\beta_{\text {post }}$ holds

Show: $I_{c} \wedge \beta_{\text {out }} \supset \beta_{\text {post }}$

Proof: st.block - st.blvl = st.blvl'+1 = st.block'+1 and st.assoc = st.assoc'.

For the function leaveblock

3. Concrete operation

Show: $\beta_{\text {in }} \wedge I_{c}\{$ body $\} A_{\text {out }} \wedge I_{c}$

Proof: st.blvl - st.blul'-1 is clear. By the for statement each $s q_{i}$ for $i \epsilon$

$[0 . . m-1]$ is adjusted by the first statement. For each of indis, del, and delall, we have the pre condition $i \in[0 . m-1]$ by the for statement.

The other part of pre of del, mbr(st.as,i,j), holds by indis. In the then 
case, del(st.as, $, j, j)$ deletes all entries in $s q_{i}$ up to but not including $j$.

Because $j$ is the first $j$ with st.info[j].b|sst.blvi<st.blvl', the block level ordering asserted by $I_{c}$ ensures $\beta_{\text {out }}$. In the else case all st.info[j].hl>st.blvl whence $s q_{i}$ should become $<>$, which delall does. Rout follows since st.info[j].bl<st.blvl' $-4 \operatorname{mbr}(s t . a s, i, j)$. In both the then and eise cases, $I_{c}$ still holds because the lists only get shorter and st.b|v|>1 on entry.

4a. $\beta_{\text {in }}$ holds

Show: $I_{c} \wedge$ st.block $>1>$ st.blvil>1

Proof: In the rep function, st.block and st.blvl correspond.

4b. $\beta_{\text {post holds }}$

Show: $I_{c} \wedge \beta_{\text {pre }} \wedge \beta_{\text {out }} \supset \beta_{\text {post }}$

Proof: Since st.blvl=st.blvl'-1, we have st.block $=$ st.block'$^{\prime}-1$ as required. By

$\beta_{\text {out }}$ and the rep function, st.assoc-st.assoc' - $\{<s, x, u i>$ st $x \geq s t . b l o c k '\}$.

For the function full

3. Concrete operation

Show: $I_{c}\{$ th-full(st.as) $\} \wedge_{\text {out }} \wedge I_{c}$

Proof: $\beta_{\text {out }}$ is exactly the post condition of full in condis. $I_{c}$ is unchanged.

4a. Bin holds

$\beta_{\text {in }}$ is true

4b. $\beta_{\text {post }}$ holds

Show: $I_{c} \wedge \beta_{\text {out }} \supset \beta_{\text {post }}$

Proof: $t=\left(\right.$ SIGMA $_{j} \in[0 . m-1]$ length $\left.\left(s . s q_{j}\right)-n\right)=($ cardinality(st.assoc) $-n)$. QED

\section{Implementation of the form Condis}

As discussed earlier, the abstract representation of condis is a set of precisely $m$ sequences of integers. The integers in these sequences are all in the range 1 to $n$, and a particular integer appears at most once in some sequence.

As one might expect, the sequences will be represented by singly linked lists. In fact we shall use an integer vector, 4 (for link-table), to store all of the lists which represent sequences in a condis. The fact that an index $i$ into It is in the $k^{\text {th }}$ position of such a list will represent the fact that $i$ appears in the $k^{\text {th }}$ position of the corresponding abstract sequence. A separate vector, $s q$, of length $m$, is used for the heads of the lists. In all cases, zero, which is not a legal condis sequence element, is used to indicate the end of a list; thus, in particular, if $s q[j]=0$, the $j^{\text {th }}$ condis sequence is emply. ${ }^{5}$ A separate list of those integers which are not 
currently members of any sequence is also maintained, and the head of this list is maintained in the simple variable free. The following diagram illustrates one possible configuration of a condis object which has been declared with $m=3$ and $n=10$ :

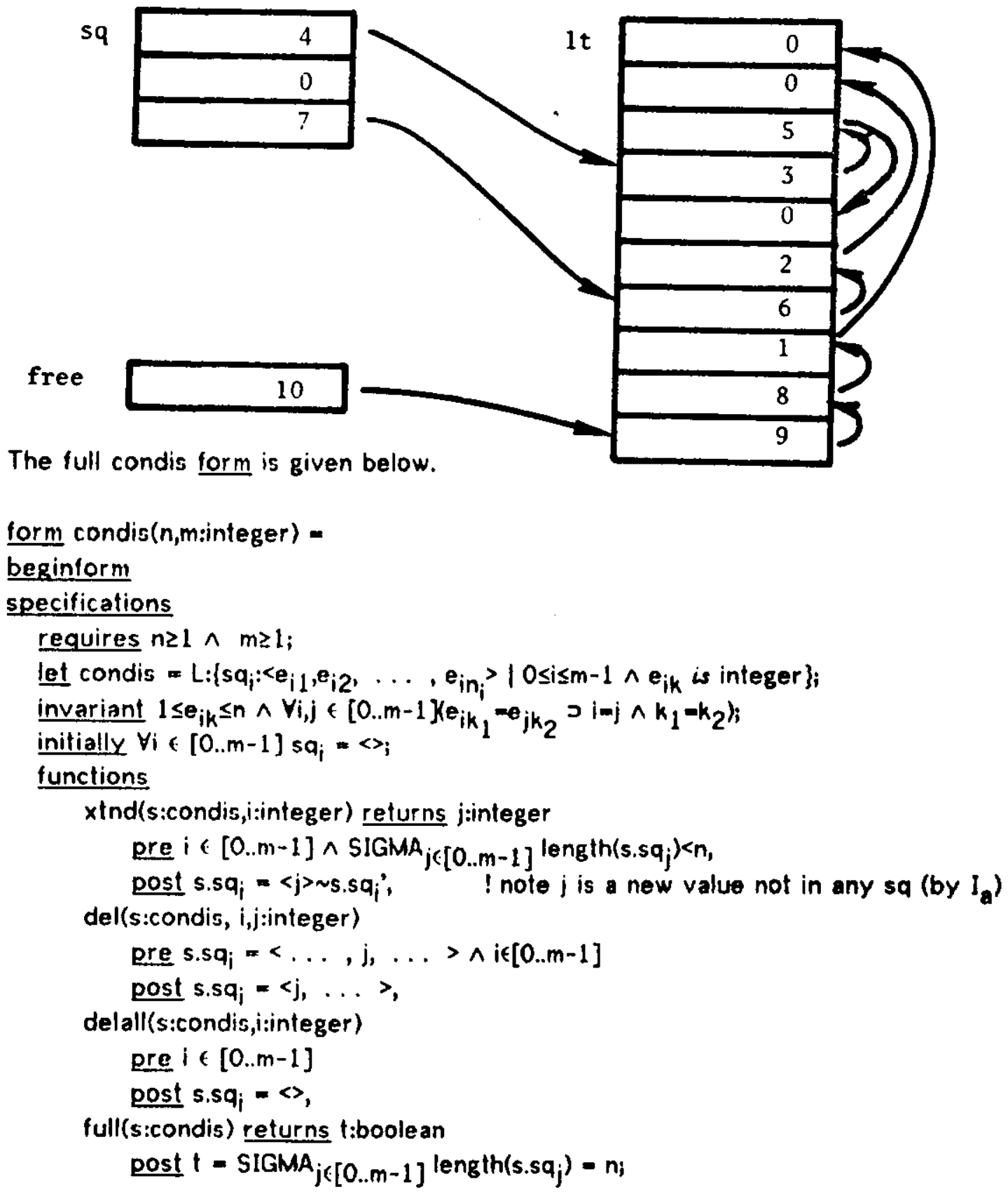

5 We can now explain why the function delall is not redundant. The knowledge that zero ends a list is private to condis, and therefore it is not known in symtab. Hence, in the body of leaveblock of symtab, the operation "delall(as,i)" cannot be replaced by "del(as,i,0)". To do so would violate the pre condition of del because if $j$ is a member of $s q_{j}$, it means $j \geq 1$. 
generator indis(s:condis,itinteger) extends x:integer

requires $0 \leq i \leq m-1$

let indis $=5.5 q_{\text {; }}$ where indis $\mu<>>$

(indis $=c \sim\langle x\rangle \sim d$ and $c,\langle x\rangle$, and $d$ are disjoint);

rule for $(l, x,<s, i>, S T)=$

premise $s . s q_{i}=c \sim<x>\sim d \wedge \mathrm{I}(c)\{S T\} \mathrm{I}(\mathrm{c} \sim<x>)_{\text {; }}$

rule first $\left(P, x,\left\langle s, i>, \beta, s_{1}, s_{2}, Q\right)=\right.$

premise $s . s q_{i} \approx c \sim<x>\sim d \wedge P \wedge \forall y\left(c(\neg \beta(y)) \wedge \beta(x)\left\{s_{1}\right\} Q\right.$,

premise $P \wedge \forall y\left(s . s q_{j} \sim \beta(y)\left\{S_{2}\right\} Q_{\text {; }}\right.$

auxiliary predicates

follows(s:condis,i,j:integer) $d f$ $3 k$ st $s q_{k}=\langle\ldots, i, \ldots, j, \ldots\rangle$,

$\operatorname{mbr}\left(s:\right.$ condis,i,j:integer) ${ }_{\mathrm{df}} \mathbf{s q}_{i}=\left\langle\ldots, \mathrm{j}_{1} \ldots>\right.$;

\section{representation}

unique

sq: vector(integer, $0, m-1$ ),

It: vector(integer, $1, n)$,

free: integer

init begin free $\leftarrow 1$; for i:upto $(1, n-1)$ do $1 t[i]+i+1 ; 1 t[n]+0$;

for i:upto $(0, m-1)$ do $s q[i]+0$ end;

$\underline{\text { rep}}(\mathrm{sq}, \mathrm{tt}$, free $)=\left\{S Q_{j} \mid 0 \leq i \leq m-1\right\}$ where

if $s q[i]=0$ then $S Q_{i}=<$ else

if $s q[i]=p_{1} \wedge\left(\forall j \in[1 . . k-1] \| t\left[p_{j}\right]=p_{j+1}\right) \wedge \| t\left[p_{k}\right]=0$ then $S Q_{i}=\left\langle p_{1}, \ldots, p_{k}\right\rangle$; invariant

$0 \leq$ tree $\leq n$

$\wedge \forall j \in[0 . . m-1] 0 \leq s q[j] \leq n$

$\wedge \forall k \in[1 . . n] 0 \leq \mathrm{It}[k] \leq n$

$\wedge\{$ free, $s q[j], 1 t[k]\}=\left\{m+1 \quad 0^{\prime} s, 1,2, \ldots, n\right\} \quad$ ! this term is a multiset equality

$\wedge \forall i \in[1 . . n](\operatorname{succ}($ free,,$i)$ xor $3 ! j(\operatorname{succ}(s q[j], i)))$

where $\operatorname{succ}(i, j) \equiv \mathrm{df} i=j \vee\left(i \neq 0\right.$ cand $\left.\operatorname{succ}\left(i t[i]_{j}\right)\right)$ i

implementation

body $x$ tnd in s.free $* 0 \wedge i \in[0 . m-1]$

out $\left(\operatorname{succ}\left(s . f r e e^{\prime}, j\right) \wedge \operatorname{succ}(s . s q[i] j) \wedge s . s q[i]=j \wedge s . t t[j]=s . s q^{\prime}[i]\right)=$ begin

$\mathrm{j} \leftarrow$ s.free; s.free $\leftarrow$ s.lt $[\mathrm{j}]$;

$s .1 t[j] \leftarrow s . s q[i] ; s . s q[i] \leftarrow j ;$

end: 
body del in succ(s.sq[i],j) $\wedge i \in[0 . . m-1] \wedge j \in[0 . . n] \underline{\text { out }}(s . s q[i]-j)-$

if $s . s q[i] * j$ then

begin local k:integer;

$k \leftarrow s . s q[i] ;$

while $s . t t[k] \neq j$ do $k \leftarrow s . t t[k]$;

$s . I t[k] \leftarrow$ s.free; s.free $\leftarrow$ s.sq[i]; s.sq[i] $\leftarrow j ;$

end;

body delall in $i \in[0 . . m-1]$ out $(s . s q[i]=0)=$

s.del $(s, i, 0) ; \quad$ a call to the concrete body del, not the abstract function del

body full out $(t=($ s.free $=0))=$

$t \leftarrow$ s.free $=0$;

formbody indis w

beginform

representation

rep $(s . s q, s . I t, i, x)=$

if $s . s q[i]=0$ then $<>$ else

if $x=0$ then $c \sim d$ where $c=s . s q_{j}$ and $d=\langle>$ else $c \sim<x>\sim d$

where $c=\left\langle p_{1}, \ldots, p_{r-1}\right\rangle, x=p_{r}, d=\left\langle p_{r+1}, \ldots, p_{k}\right\rangle$,

invariant true;

$p_{1}=s . s q[i]$ s.lt $\left[p_{k}\right]=0$, and $\left(Y j \in[1 . . k-1] s . I t\left[p_{j}\right]=p_{j+1}\right) ;$

implementation

body \&init out $(x=5.5 q[i] \wedge(\& b=5 . s q[i] \mu 0))=$

$(x \leftarrow s . s q[i] ; \& b \leftarrow x \neq 0) ;$

body \&next in $\operatorname{succ}(s . s q[i], x) \wedge x \neq 0$ out $\left(x=s .1 t\left[x^{\prime}\right] \wedge\left(\& b=s . \operatorname{lt}\left[x^{\prime}\right] \wedge 0\right)\right)=$

$(x \leftarrow s .1 t[x] ; \& b+x \neq 0) ;$

endform

endform

The implementation of the four operations in condis should be fairly obvious. $x$ tnd merely removes an entry from the free list and places it at the head of the appropriate list; note that this entry is returned (in j) as the value of function $x$ tnd. del is a bit more interesting. It searches the appropriate list for the entry in It which points to the first entry, $j$, which is not to be removed. It then moves the entire initial portion of the list to the free space list by simply setting the proper pointers. If all the entries are to be removed, delall does this; it calls del to search for the list-ending zero and to move the entire list to the free space list. full just tests if the free space list is empty. 
The predicate succ defined in the concrete invariant is closely related to the abstract predicate follows. Although the parameterizations of the two predicates are different, they ask the "same" question and are related by

$$
\text { follows (rep(sq,it,free), } i, j)=\operatorname{succ}(i, j)
$$

The form indis(s,i) defines a generator for elements of the integer sequence $s_{j}$, starting with first $\left(s_{j}\right)$. Abstractly, an indis is composed of three (sub)sequences, the first containing the elements already generated, the second the (singleton) current element, and the third the other elements yet to be seen.

In [Shaw76b] we discussed the proof rules for iteration statements. We showed that certain simplifying assumptions about the generator can yield simple proof rules; these assumptions are satisfied by indis, as we will show in the verification of condis. We therefore have a proof rule for the for statement which corresponds closely to Hoare's sequence rule and also a proof rule for the first statement. These proof rules are given in the specifications of indis, and indeed constitute the major part of those specifications. The basis for this specification technique for generators is given in [Shaw 76b]

\section{Verification of Condis}

We can now verify the form condis.

For the form

1. Representation validity

Show: $l_{c}(s q$, it, free $)>l_{a}$ (rep(sq,It,free))

Proof: $1 \leq e_{i k} \leq n$ holds by the bounds on $s q[j]$ and $I t[k]$ and the fact that the rep function drops the zeroes that indicate the end of a list. The ik's are distinct because the multiset \{sq[j], It[k]\} contains each of $1,2, \ldots$, $n$ at most once. The multiset property of $I_{c}$ implies succ(free,0) and $\operatorname{succ}(s q[j], 0)$.

2. Initialization

Show: $n \geq 1 \wedge m \geq 1$ ( init $\} \forall i \in[0 . m-1] s q_{j} \approx<>\wedge \mathbb{l}_{c}$

Proof: After init we have free $=1,1 t[1]=2, \ldots, I t[n-1]=n, I t[n]=0, s q[0]=0, \ldots$, $s q[m-1]=0$. Using the rep function, each $s q_{j}=<>$ since each $s q[i]=0$. $n \geq 1$ means $0 \leq$ frees $n$. The bounds on $s q[j]$ and $I t[k]$ and the multiset property are clear. $Y i \in[1 . . n](\operatorname{succ}(f r e \theta, i) \wedge \sim \operatorname{succ}(0, i))$. 
For the function $x$ tnd

3. Concrete operation

Show: s.free $0 \wedge i \in[0 . m-1] \wedge I_{c}\{$ body $\} \beta_{\text {out }} \wedge I_{c}$

Proof: The four terms of $\beta_{\text {out }}$ are clear as are the bounds in $I_{c}$. The multisef property holds because the body permutes the values s.free', s.sq'[i], and s.lt'[s.free']. Since the head of s.free moves to the head of s.sq[i], each $i \in[1 . . n]$ still satisfies exactly one succ term. $\beta_{\text {in }}$ (and $I_{c}$ ) ensures that the accesses to s.tt and s.sq are within bounds.

4a. $\beta_{\text {in }}$ holds

Show: $J_{c} \wedge \beta_{\text {pre }} \supset \beta_{\text {in }}$

Proof: $i \in[0 . . m-1]$ is immediate. If s.free $=0$, then the multiset property of

$I_{c}$ means, using the rep function, that the SIGMA term is exactly $n$, a contradiction. Hence s.freepo.

4b. $\beta_{\text {post }}$ holds

Show: $I_{c} \wedge \beta_{\text {out }} \wedge \beta_{\text {pre }}>s q_{j}=<j>\sim s q_{i}$

Proof: Since s.sq[i]wi and s.It $[j]-s . s q^{\prime}[i]$, the rep function gives $s q_{j}=<j>\sim s q_{j}$.

For the function del

3. Concrete operation

Show: $\beta_{\text {in }} \wedge I_{c}\{$ body $\} \mathrm{s.sq}[i] \times x j \wedge I_{c}$

Proof: If s.sq[i]xi then $\beta_{\text {out }}$ holds and $I_{c}$ is unchanged. If s.sq[i]pj then define the set $G_{p}=\{x \mid \operatorname{succ}(s . s q[i], x) \wedge \operatorname{succ}(x, p)\}$. Add the ghost operation "H$+H \cup\{k\}$ " after "k-s.It[k]" in the while loop and add "Hk-\{k\}" after " $k-5.5 q[i] "$. A while-loop invariant (placed before the test) is then $H=G_{k}$ because $G_{s . s q[i]}=\{s . s q[i]\}$ and

$$
H=G_{k} \wedge \text { s.lt }[k] \vDash j>H \cup\{s . I t[k]\}=G_{s . I t[k]}
$$

The while terminates because succ(s.sq[i],j) and s.sq[i]; $j$. At termination s.It $[k]=j$ and $H=G_{k}$. The multiset property of $I_{c}$ holds because the last three statements in the body permute the values s.free', s.sq'[i], and s.lt'[k] Furthermore, each element in $H$ is now a successor of s.free rather than of s.sq[i] All other successors of s.sq[i] and all previous successors of s.free remain so, respectively. $\beta_{\text {out }}$ and the bounds in $l_{c}$ are clear.

4a. $\beta_{\text {in }}$ holds

$$
\text { Show: } I_{c} \wedge \beta_{\text {pre }} \supset \beta_{\text {in }}
$$

Proof: Immediate from $\beta_{p r e}$ and $I_{a}$ for condis.

4b. $\beta_{\text {post }}$ holds

Show: $\mathrm{l}_{c} \wedge \beta_{\text {pre }} \wedge \beta_{\text {out }}>s q_{i}=<j, \ldots>$

Proof: Only $s q_{i}$ changes. $s q_{j}$ now begins with $j$ and there are no other changes to $s q_{j}$. 
For the function delall

3. Concrete operation

Show: $\beta_{\text {in }} \wedge I_{c}\{$ s.del $(s, i, 0)\}$ s.sq[i] $=0 \wedge I_{c}$

Proof: $\beta_{\text {in }}$ and the multiset property of $I_{c}$ imply in holds for s.del. ( $I_{c}$ holds for s.del as required.) The out for s.del gives s.sq[i]=0. $I_{c}$ after $s$.del gives $I_{c}$ after delall.

4a. Bin $_{\text {in }}$ holds

Show: $i \&[0 . . m-1] \supset$ i $[$ [0..m-1]

Proof: Immediate

4b. $\beta_{\text {post }}$ holds

Show: $I_{c} \wedge i\left([0 . m-1] \wedge s . s q[i]=0 \supset s q_{i}=<>\right.$

Proof: Only $s q_{i}$ changes. s.sq[i] $=0$ means $s q_{i} \approx<>$.

For the function full

3. Concrete operation

Show: $I_{c}\{$ t-s.free $=0\} t=($ s.free $=0) \wedge I_{c}$

Proof: Immediate

4a. $B_{\text {in }}$ holds

$\beta_{\text {in }}$ is true

4b. $\beta_{\text {post }}$ holds

Show: $I_{c} \wedge \beta_{\text {out }} \supset \beta_{\text {post }}$

Proof: $t=($ s.free $=0)=(\operatorname{SIGMA} \ldots=n)$ using the multiset property of $I_{c}$.

To verify the indis generator, we must first reconstruct the pre and post conditions from the specified proof rules:

Sinit

post $\left(\& b=s . s q_{j} \neq<>\right) \wedge\left(\& b \supset x=\right.$ first $\left(s . s q_{j}\right) \wedge c=\langle>)$

\&next

pre $\operatorname{mbr}(s, i, x)$

post $\left(\& b=d^{\prime} \neq<>\right) \wedge\left(\& b \supset x=f i r s t\left(d^{\prime}\right) \wedge c=c^{\prime} \sim<x^{\prime}>\right)$

Next, we must show that indis satisfies the standerd aggregate essumptions:

(a) The indis abstraction is explicated in terms of sequences. The normal empty sequence (<>), concatenation operator $(\sim)$, and leading element selector (first) are available.

(b) The complete sequence to be generated is $s . s q_{j}$, which can be decomposed as indicated in the let clause of indis. 
(c) The specifications of \&init and \&next have the required form.

Furthermore, indis satisfies the basic generator assumptions because (a) \&init and \&next terminate and (b) \&init and \&next alter only the indis variable $x$ (and the return value \&b).

Since "sq", "It", and "free" are unchanged by indis, the $I_{c}$ of condis still holds and will be used in the proof.

For the form (indis)

1. Representation validity

Show: $I_{c} \supset I_{a}$, i.e., true $\supset$ true

Proof: Immediate

2. Initialization

Show: $0 \leq i \leq m-1\{\}$ true $\wedge$ true

Proof: Immediate

For the function \&init

3. Concrete operation

Show: true $\{x+5.5 q[i\} \& b+x \neq 0\} x-5 . s q[i] \wedge(\& b=s . s q[i] \wedge 0)$

Proof: Clear

4a. $\beta_{\text {in }}$ holds

$\beta_{\text {in }}$ is true

4b. Bpost holds

Show: $x=s . s q[i] \wedge(\& b \equiv s . s q[i] \wedge 0) \supset$

$\left(\& b=s . s q_{j} \neq<>\right) \wedge\left(\& b>x=f i r s t\left(s . s q_{j}\right) \wedge c=\langle>)\right.$

Proof: From the rep function for indis, $s . s q_{i}=$ (if s.sq[i]=0 then $<$ else

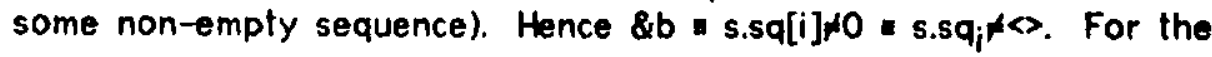
second term of the conclusion, assume \&b. Then $x-s . s q[i] \geqslant 0$ and the final clause of rep gives $5 . s q_{i}=c \sim<x>\sim d$. Since $x-s . s q[i] m p_{1}$, then $c=$ <> whence also $x=$ first $\left(5 . s q_{j}\right)$.

For the function \&next

3. Concrete operation

Similar to Rinit.3

4a. $\beta_{\text {in }}$ holds

Show: $\operatorname{mbr}(s, i, x)>\operatorname{succ}(s . s q[i] x) \wedge x \neq 0$

Proof: $\operatorname{mbr}(s, i, x)$ means $x \neq 0$ by $l_{a}$ for condis. The term $\operatorname{succ}(s . s q[i] x)$

follows from $\operatorname{mbr}(s, i, x)$, the rep function, and the definition of succ.

4b. Bpost holds

Show: $m b r\left(s, i, x^{\prime}\right) \wedge x=5.1 t\left[x^{\prime}\right] \wedge\left(\& b=s .1 t\left[x^{\prime}\right] \neq 0\right) \supset$

$\left(\& b=d^{\prime} \wedge<>\right) \wedge\left(\& b \supset x=\right.$ first $\left.\left(d^{\prime}\right) \wedge c-c^{\prime} \sim\left\langle x^{\prime}\right\rangle\right)$ 
Proof: $m b r\left(s, i, x^{\prime}\right)$ means $x^{\prime} \neq 0$ and $s . s q ;<>$, and therefore by the rep function also s.sq[i] $\neq 0$. Hence in the final clause of the rep function, $\& b$ a. It $\left[x^{\prime}\right] * 0 \approx d^{\prime} \neq\langle>$. For the second term of the conclusion, assume \&b. Then $x=s .1 t\left[x^{\prime}\right] \times 0$ and the final clause of rep gives s.sq; $-c r<x>n d$ and, because $x^{\prime}>0$, also $s . s q_{i}=c^{\prime} \sim\left\langle x^{\prime}>\sim\left\langle d^{\prime}>\right.\right.$. Since $x=s .1 t\left[x^{\prime}\right]$, it follows that $x=$ first (d') and $c=c^{\prime} \sim\left\langle x^{\prime}>\right.$.

\section{Examples of the Use of Symtab}

In this section we shall present a skeletal example which involves three different styles of usage of the symtab abstraction. It is not our intent either to make this example complete or to suggest that the utility of the abstraction is limited to these three cases. Rather, we wish to bolster the reader's intuition about ways in which the abstraction might be used.

The example we have chosen is a multi-pass compiler for an Algol-like (i.e., blockstructured) language, and indeed we have restricted ourselves to the first two passes -lexical and syntactic analysis, respectively. In this scheme, the first pass is responsible for reading units of the source file (identifiers, literals, punctuation marks, etc.) and converting them to an internal form called a "lexeme". These lexemes are written onto a file which will be read again by the second pass. The second pass is responsible for reading the file of lexemes generated by the first pass and performing syntactic analysis. Although it is not important to our example, the output of the second pass will likely be some other intermediate representation (e.g., reverse polish or trees) which is suitable for optimization and code generation.

Here, then, is the skeletal program; more detailed comments on the uses of the symtab abstraction, and on the program in general, follow the example.

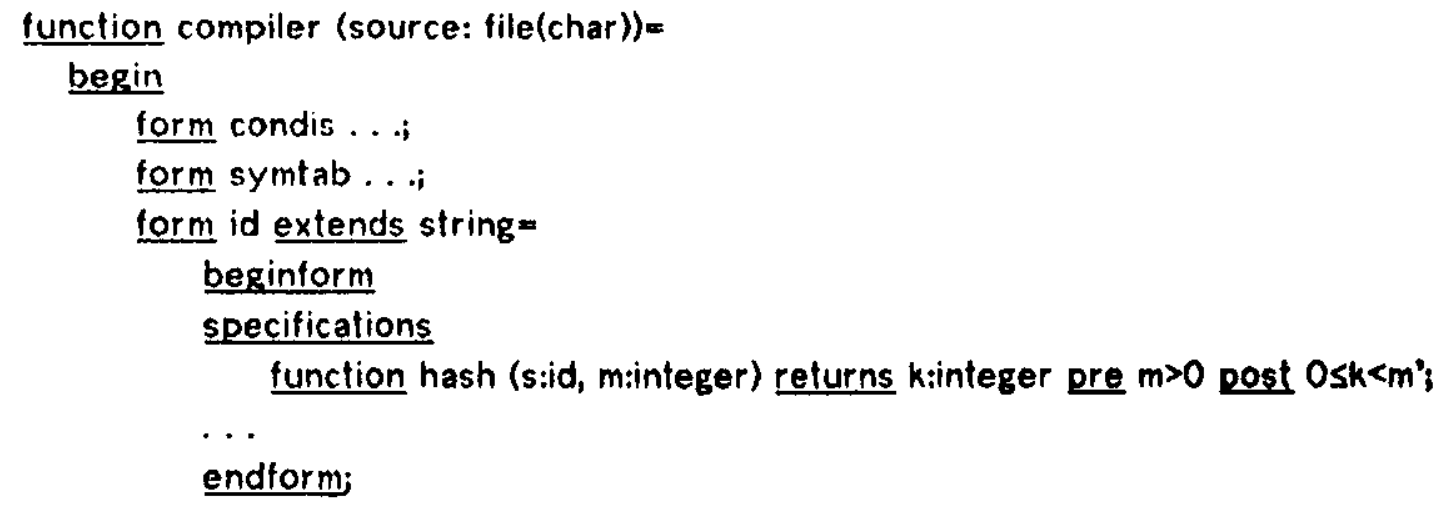




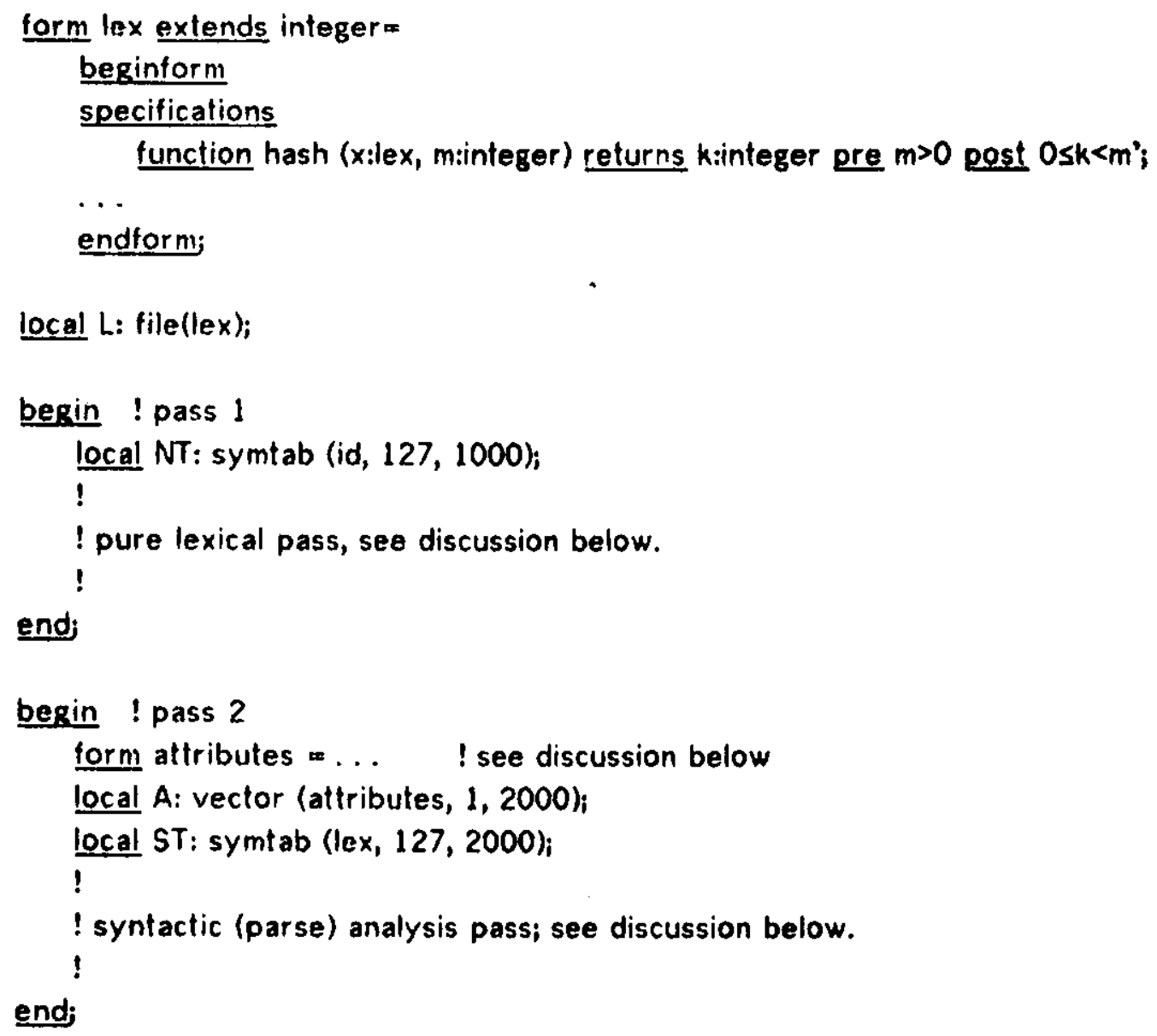

This program first defines four forms. Symtab and condis have been defined in detail proviously and hence are not repeated. The forms id and lex are extensions of strings and integers, respectively, and merely add hashing functions; we have not defined the implementations of these functions, since they are not germane to the example. Note too that a file of lexes is defined at the outermost block level; this file is the explicit interface between the first and second passes.

As noted earlier, the function of the first pass is to convert the external representation of the program (a file of characters) into a more convenient internal form -- namely a file of lexemes (where each lexeme represents an atom of the language). Since this pass does no syntactic analysis, in particular it does not recognize block structure. This implies that all occurrences of the same atom (e.g., "xyz") will be mapped to the same lexeme. This mapping is accomplished through the use of the NT (for name-table) instantiation of symtab; indeed, the only use of NT is to obtain this unique mapping and the instantiation is therefore deleted on exit from the block in which the first pass is accomplished. 
In skeletal form, the body of the block for pass 1 might look somewhat as follows:

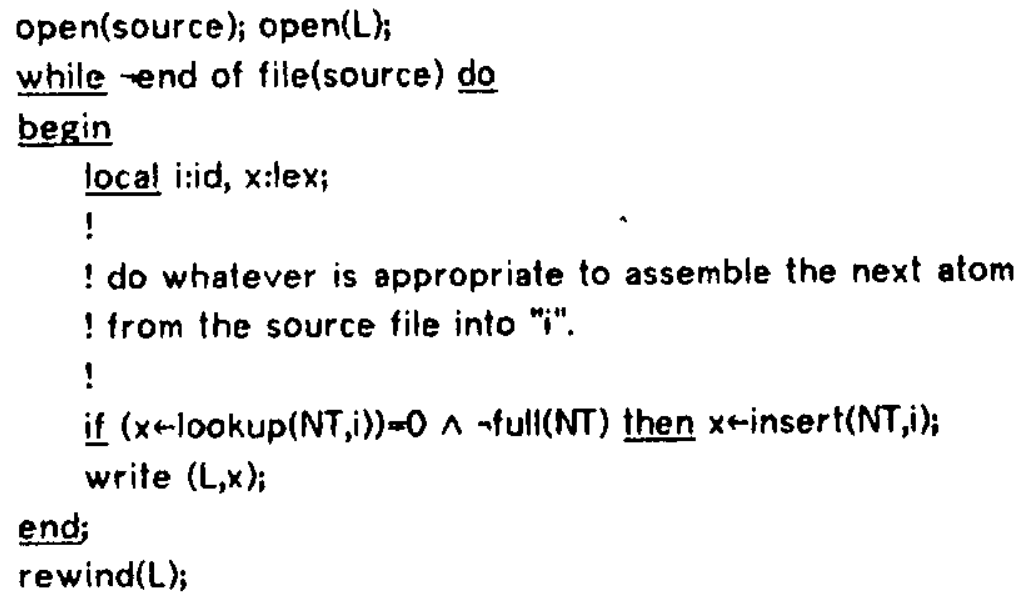

Note that the operations enterblock and leaveblock are not used, all insert operations are done at the same block level, and only one entry per atom will be made.

The second pass is substantially more complex since it performs the full syntactic analysis; hence we will not even attempt to illustrate its skeletal form. We would, however, like to point out several things about it.

First, notice that this block defines a form named attributes. We have not shown the body of this form, since it will be highly language- and machine-specific. However, the notion is that this form provides for the storage and manipulation of whatever information must be retained about a symbol, e.g., its type, run-time storage address, array bounds, and so forth.

Second, we have declared a vector, A, of these attribute objects. As suggested in an earlier section, instances declared at a given block level will be associated with unique integer, but this integer will be different from the one associated with the same identifier declared at a different block level. These integers will, in turn, be used as indices into tho vector A (e.g., to set and retrieve information about the identifier).

Finally, we have declared another instantiation of symtab, ST. This one will be used to recognize block structure, and, specifically, will map from the simple lexemes generated in the first pass into indices into the vector, A, of attributes. As the parser detects blocks (beginend pairs) in the source program, it will invoke enterblock and leaveblock. The declaration processing routines will invoke defined to determine whether an identifier has been declared twice at the same block level (presumably an error), and perform insert operations to define the instances of the identifier at the current block level. The rest of the compiler will perform lookup operations to obtain the index of the attribute vector entry associated with specific lexemes. (Note, by the way, that by appropriate ordering of insert and lookup operations the declaration processor can obtain either of the interpretations of "block-structure" discussed in the introduction.) 
Before leaving this example, let us return to the form attributes (defined in pass 2) to illustrate another potential use of the symtab abstraction. As was mentioned in the introduction, in general the mapping from identifier to unique integer may be contextsensitive. Block structure is the most familiar form of such sensitivity, but another is name qualification, as in field selectors for records. In many languages one makes a declaration such as

$x:$ record(name:string, age:integer, $z$ :integer);

and then refers to "x.name", "x.age", and "x.z". A problem arises when, at the same block level, there is another declaration such as

$$
\text { y:record(ss:integer, z:boolean); }
$$

In such a case the identifier " $z$ " is no longer unique -- its interpretation depends upon the name it qualifies.

There are many ways one might treat this, including inserting each of " $x$ ", "x.name", "x.age", "x.z", " $y "$, "y.ss", and "y.z" as complete identifiers in ST. An attractive alternative, however, is to include instantiations of symtab in each of the attributes; that is, to make form attributes appear somewhat as follows:

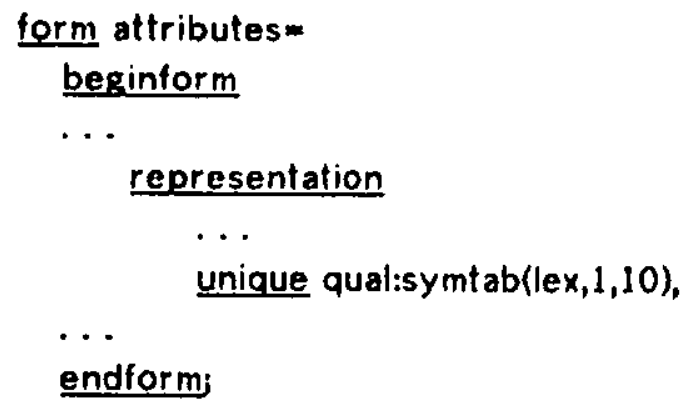

If this is done, then to determine the interpretation of " $x . z^{n}$ one would first search ST for the index, $i$, associated with the lexeme for " $x$ ", then search $A[i] q u a l$ for the index associated with the lexeme for " $z$ ".

Although this compiler example has been sketchy, we hope that it has suggested some of the ways in which the symtab abstraction may be applied. The details of the example are not important, except insotar as they help the reader's intuition; what is important is the notion that well-chosen abstractions have many uses. The class of broadly useful abstractions is simply too large to include them all in a single programming language -- hence Alphard has chosen to provide a linguistic facility so that the programmer may define them. Many such (verified) abstractions will find their way into the library, and hence incrementally enhance the "power" available to the programmer -- without, at the same time, limiting him to the language 
designer's preconceived notions of what constitutes an appropriate set of abstractions (or, for that matter, implementations).

\section{Conclusions}

A programming language is a fool for the construction and communication of prograns; as such its utility should be measured relative to these tasks. In other words, the language should be used, and the quality of that use must be judged. While this is true of any programming language, it is especially so of one such as Alphard, which departs substantially from those in common use.

Thus, in this and other reports we are attempling to exhibit Alphard in relatively realistic contexts and, along with the reader, to judge the practical utility of our creation. It is far too soon to draw definitive conclusions -. that must await the use of Alphard in real programs -- but we would like to share some of our impressions resulting from these experiences.

First, the symtab abstraction is about the (conceptual) size we envision for most abstractions; larger programs will be constructed by further "layering". Thus we take our ability to specify and verify this form as fairly strong evidence that larger programs will also be tractable.

Second, in most respects the implementation is a practical, efficient one. This reintorces our intuitions that no efficiency need be sacrificed to obtain clear, verifiable programs. (The one exception to this statement is our use of fixed-sized vectors and, correspondingly, integers for the unique identification of symbols. A more realistic implementation would, perhaps, have done true dynamic storage allocation and used references. We avoided this implementation primarily because it would have carried us into portions of Alphard not covered in previous reports, but also because those portions of the language are still in flux. We trust that the reader will forgive this departure from realism.)

Third, one of the anticipated advantages of an Alphard-like language is that a library of verified abstractions will develop. Both of the forms developed here might well go into that library so we are getting some evidence that this hoped-for advantage will be realized.

Fourth, one of our private objectives was to make the form mechanism strong enough to support an extremely broad class of abstractions -- the ultimate target being the spectrum covered by our intuitive notion of the word "abstraction". The evidence is not conclusive, but we are feeling better about meeting that goal all the time.

Finally, we should say a few words about our experience concerning the effort needed 
to define a form. It should be clear that the actual code in a form body, i.e., the implementation part, is roughly the same size as the corresponding code in other languages (although the first statement does seem to shorten many of the examples). Moreover, for some reason, the information needed for verification (abstract and concrete invariants, abstract pre and post conditions, rep function, etc.) usually seems about equal to the code size; thus a full form is about twice the size of the code alone. This does not particularly concern us, since these kinds of specifications tend to replace much of the documentation that would otherwise be needed -- and they are certainly more precise.

We find the verification of a form, once the specifications and code have been written, to be more difficult and time-consuming than coding, but not unreasonably so (say by as much as a factor of two or three). Sometimes it is necessary to modify the specifications, or the code, during the verification in order to remove inconsistencies that are uncovered. The verification may also suggest different specifications, usually ones that are more constrained but sometimes simpler ones. In spite of the difficulties, the bodies of functions tend to be small and their proofs correspondingly small, as can be seen from these examples. Moreover, the proofs of the two torms symtab and condis were independent. To date our proofs have been manually generated, but we envision having automated, interactive aids in the future. These should reduce the verification time to approximately the coding time. Since this is less than the time currently spent on debugging, we feel highly encour aged.

The majority of our time goes into designing and specifying the abstraction. There are two related aspects of this: getting the intuitive abstraction "right", and formalizing it (at least sufficiently for it to be verified). The two appear related in that difficulty in formalizing an intuitive abstraction often seems to uncover muddy thinking at the intuitive level. While we seem to be improving our ability to formalize, indicating that it is a learnable skill, we have no easy rules for picking the right abstraction in the first place. While, with practice, our abilities in choosing abstractions may also improve, we suspect that this is fundamental problem of design and has a significant aesthetic component.

It is clear that we are just learning to use the power of the tools we are creating and exploring. Much remains to be discovered about what is possible or impossible, easy or hard, and reasonable or unreasonable to do with the facilities. In this connection we note that an early version of symtab was a one-level form used no generator such as indis, and had only some of the same verification information. Although that version of symtab used the same implementation ideas, it was essentially incomprehensible. When we realized that multiple ideas were becoming confused, we separated the maintenance of the lists from the lookup algorithms. The result was that the code, the specifications, and the verification all became much more manageable.

\section{Acknowledgements}

We owe a great dr:- is our colleagues at CMU and ISI, especially Mario Barbacci, Neil 
Goldman, Donald Good, John Guttag, Paul Hilfinger, David Jefferson, Anita Jones, David Lamb, David Musser, Karla Perdue, Kamesh Ramakrishna, and David Wile. We would also like to thank James Horning and Barbara Liskov and their groups at the University of Toronto and Massachusetts Institute of Technology, respectively, for their critical reviews of Alphard.

\section{References}

[Gries:71] David Gries, Compiler Construction for Digital Computers, Witey, 1971.

[Guttag76] Jolnn Guttag, "Abstract Data Types and the Development of Data Structures", Supplement to the Proceedings of the SICPLAN,SICMOD Conference on Datn: Ahstraction, Definition, and' Structure, March 1976 (pp. 37-46). Also Communications of the $A C M$ (to appear).

[Halmos60] Paul R. Halmos, Nuive Set Theory, Van Nostrand, 1960.

[Hoare72] C. A. R. Hoare, "Proof of Correctness of Data Representations", Acte Informatica, 1, 4, 1972 (pp. 271-281).

[Knuth73] Donald E. Knuth, The Art of Computer Programming, Volume 3, Sorting and Searching, Addison-Wesley, 1973.

[Shaw76a] Mary Shaw, "Abstraction and Verification in Alphard: Design and Verification of a Tree Handiler", Proc. Fish Texas Conference on Computing Systems, 1976 (pp. 86-94).

[Shaw76b] Mary Shaw, Wim. A. Wulf, and Ralph L. London, "Abstraction and Verification in Alphard: Ileration and Generators", Carnegie-Mallon University and USC Information Scionces Institute Technical Reports, 1976. Also Communications of the ACM (to appear).

[Wegbreit76] Ben Wegbreit and Jay M. Spitzen, "Proving Properties of Complex Data Structures", Journal of the AC.M, 23, 2, April 1976 (pp. 389-396).

[Wulf76a] Wm. A. Wulf, Ralph L. London, and Mary Shaw, "Abstraction and Verification in Alphard: Introduclion to Language and Mathodology", Carnegie-Mollon University and USC Information Sciences Institute Technical Roports, 1976.

[Wulf 76b] Wm. A. Wulf, Ralph L. London, and Mary Shaw, "An Introduction to the Construction and Verification of Aiphard Programs", IEEE Transactions on Software Engineering, SE2, 4, December 1976 (pp. 253-265). 


\section{Appendix A \\ Informal Description of Verification Methodology}

Alphard's verification methodology is designed to determine whether a form will actually behave as promised by its abstract specifications. The methodology depends on explicitly separating the description of how an object behaves from the code that manipulates the representation in order to achieve that behavior. It is derived from Hore's technique for showing correctness of data representations[Hoare 72]

The abstract object and its behavior are described in terms of some mathematical entities natural to the problem domain. Graphs are used in [Shaw76a] to describe binary trees; sequences are used in [Wulf $76 a, b]$ to describe queues and stacks and in condis to describe list processing, and so on. We appeal to these abstract types

- in the invariant, which explains that an instantiation of the form may be viewed as an object of the abstract type that meets certain restrictions,

- in the initially clause, where a particular abstract object is displayed, and

- in the pre and post conditions for each function, which describe the effect the function has on an abstract object which satisfies the invariant.

The form contains a parallel set of descriptions of the concrete object and how it behaves. In many cases this makes the effect of a function much easier to specify and verify than would the abstract description alone.

Now, atthough it is useful to distinguish between the behavior we want and the data structures we operate on, we also need to show a relationship that holds between the two. This is achieved with the representation function rep $(x)$, which gives a mapping from the concrete representation to the abstract description. The purpose of a form verification is to ensure that the two invariants and the rep $(x)$ relation between them are preserved.

In order to verify a form we must therefore prove four things. Two relate to the representation itself and two must be shown for each function. Informally, the four required steps are 6 :

6 We will use $I_{a}(r e p(x))$ to denote the abstract invariant of an object whose concrete representation is $x, I_{c}(x)$ to denote the corresponding concrete invariant, italics to refer to code segments, and the names of specification clauses and assertions to refer to those formulas. In step $4 b$, "pre $\left(r e p\left(x^{\prime}\right)\right)$ " refers to the value of $x$ before execution of the function. A complete development of the form verification methodology appears in [Wulf $76 a, b]$ 
For the form

1. Representation validity

$$
I_{c}(x)>I_{a}(\operatorname{rep}(x))
$$

2. Initialization

requiress \{ init clause \} initially $(r e p(x)) \wedge J_{C}(x)$

For each function

3. Concrete operation

$i n(x) \wedge I_{c}(x)\{$ function body $\}$ out $(x) \wedge I_{c}(x)$

4. Relation between abstract and concrete

4a. $I_{c}(x) \wedge \operatorname{pre}(r e p(x))>\underline{i n}(x)$

4b. $I_{c}(x) \wedge \operatorname{pre}\left(r e p\left(x^{\prime}\right)\right) \wedge$ out $(x) \supset \operatorname{post}(\operatorname{rep}(x))$

Step 1 shows that any legal state of the concrete representation has a corresponding abstract object (the converse is deducible from the other steps). Step 2 shows that the initial state created by the representation section is legal. Step 3 is the standard verification formula for the concrete operation as a simple program; note that it enforces the preservation of $I_{c}$. Step 4 guarantees (a) that the concrete operation is applicable whenever the abstract pre condition holds and (b) that if the operation is performed, the result corresponds properly to the abstract specifications. 